\title{
In situ generation of 3D graphene-like networks from cellulose nanofibres in sintered ceramics
}

\author{
Andraž Kocjan *a, Rainer Schmidt ${ }^{b}$, Ana Lazar ${ }^{a d}$, Jesús Prado-Gonjal $^{e}$, Janez \\ Kovač $\check{c}^{f}$, Manca Logar ${ }^{g}$, Francisco J. Mompean ${ }^{c}$, Mar Garcia-Hernandez ${ }^{c e}$, Eduardo \\ Ruiz-Hitzky $^{h}$ and Bernd Wicklein ${ }^{*}$ \\ a'Department for Nanostructured Materials, Joǵef Stefan Institute, Jamova 39, 1000 Ljubljana, \\ Slovenia. E-mail: a.kocjan@ijs.si \\ ${ }^{\mathrm{b}}$ Facultad de Ciencias Físicas, GFMC, Universidad Complutense de Madrid, 28040 Madrid, Spain \\ 'Laboratorio de Heteroestructuras con aplicacion en spintronica, Unidad asociada UCM/CSIC, 28049 \\ Madrid, Spain \\ dJoǵef Stefan International Postgraduate School, Jamova 39, 1000 Ljubljana, Slovenia \\ eMaterials Science Factory, Instituto de Ciencia de Materiales de Madrid (ICMM), Consejo Superior \\ de Investigaciones Científicas (CSIC), Sor Juana Inés de la Cruz 3, 28049 Madrid, Spain \\ fDepartment for Surface Engineering and Optoelectronics, Joǵef Stefan Institute, Jamova 39, 1000 \\ Ljubljana, Slovenia \\ ${ }^{g}$ Department of Materials Chemistry, National Institute of Chemistry, 1000 Ljubljana, Slovenia \\ ${ }^{\mathrm{h}}$ Department of New Architectures in Materials Chemistry, Instituto de Ciencia de Materiales de \\ Madrid (ICMM), Consejo Superior de Investigaciones Científicas (CSIC), Sor Juana Inés de la Cruz \\ 3, 28049 Madrid, Spain. E-mail: bernd@icmm.csic.es
}

Establishing a 3D electrically percolating network in an insulating matrix is key to numerous engineering and functional applications. To this end, using hydrophobic carbon nanofillers is tempting, but still results in suboptimal performance due to processing challenges. Here, we demonstrate how natural cellulose nanofibres can be in situ transformed into graphene-like sheets connected to a 3D network enhancing both the transport and the mechanical properties of sintered engineering ceramics. The network architecture also permits the decoupling of electrical and thermal conductivities, which represents a major obstacle in attaining efficient thermoelectric materials. We foresee that our transferable methodology can pave the way for the use of natural nanofibres to unravel the full potential of $3 \mathrm{D}$ graphene-like networks to accelerate development in fields like energy and telecommunications. 


\section{Introduction}

3-Dimensional (3D) percolating networks are ubiquitous in nature and technology like neural networks ensuring the communication of electrical signals $\stackrel{1}{ }$ or as macroscopic carbon architectures for sensing, healthcare and energy applications. ${ }^{2 i 5}$ Similar percolating networks in dielectric ceramic matrices can be established by adding small amounts of carbon nanofillers that provide electrical conductivity while preserving or even improving mechanical properties. ${ }^{6 / 8}$ On the other hand, phonon barriers constituted by electroconducting carbon nanotubes and other nanoscale materials can greatly reduce their thermal conductivity, ${ }^{2}$ which could improve thermoelectric materials. ${ }^{10}$ However, a major challenge represents the reliable and effective embedding of fine carbon nanofillers in ceramic matrices ${ }^{1.1 .12}$ and therefore, unconventional but facile approaches for the creation of percolating 3D networks are still sought. To date electro-conductive fillers like nanostructured carbon allotropes, i.e.carbon nanotubes, graphene, and reduced graphene oxide, are incorporated into ceramic matrices by mixing and blending processes, $\frac{1.14}{14}$ but the homogeneous dispersion of these carbon nanofillers in a ceramic matrix remains an issue attributed to their hydrophobic nature and concomitant colloidal instability in hydrophilic media. ${ }^{13.15}$ As a result, electrical and mechanical properties deteriorate or have to be compensated by increasing the amount of the filler $(>10 \%)$.

Here, we report an alternative methodology for the generation of embedded 3D conducting networks in conjunction with the use of natural nanofillers that are of high current research interest. ${ }^{1618}$ Wood-derived cellulose nanofibres $(\mathrm{CNF})$ are flexible, semicrystalline fibrils displaying an $I_{6}$ crystal structure. They are of approximately $3 \mathrm{~nm}$ thickness and exhibit extraordinary mechanical strength, optical transparency, and chemical versatility. ${ }^{1920}$ It has also been shown that $\mathrm{CNF}$ can be a versatile precursor for a variety of highly conducting carbon nanostructures produced by pyrolysis. ${ }^{21.22}$ Nonetheless, it has never been reported before that CNF could serve concomitantly as a mechanical and electrical reinforcement agent in sintered ceramics.

In the work presented, we demonstrate that CNF can be in situ converted during a spark plasma sintering (SPS) process into 3-dimensionally connected graphene-like sheets to effectively enhance the electrical and mechanical properties of engineering ceramics. The $2 \mathrm{D}$ graphene-like nanostructures were produced in dielectric alumina $\left(\mathrm{Al}_{2} \mathrm{O}_{3}\right)$ and ion conducting 
3 mol\% yttria-stabilised zirconia (YSZ) to showcase the process. The generated few-layer graphene (FLG) network not only enhanced the permittivity of the composite ceramic materials, which is relevant for telecommunication devices, but also decoupled thermal and electrical conductivities, which is of fundamental importance for engineering efficient thermoelectric materials. Lastly, we demonstrated that the FLG-ceramics were also suited for electro-discharge machining, a post-sinter shaping process for complex shapes.

\section{Results and discussion}

Fig. 1a shows an AFM micrograph of TEMPO-mediated oxidized CNF revealing a fibril thickness of $3 \mathrm{~nm}$ and a length of $400 \mathrm{i} 1000 \mathrm{~nm}$. The fibrils display the typical kinks that impart the flexibility to CNF. Dispersing CNF hydrogels in aqueous slurries of YSZ and $\mathrm{Al}_{2} \mathrm{O}_{3}$ powders rendered the respective ceramic suspensions with a $\mathrm{CNF}$ content ranging from 0.5 to $4 \mathrm{wt} \%$. Additional attrition milling results in homogeneous and colloidally stable CNFï ceramic slurries without particle segregation or de-mixing phenomena (Fig. S1À), which can be attributed to the hydrophilic nature of CNF. ${ }^{20}$ Freeze-dried CNFï ceramic composite powders contained non-aggregated, individual cellulose nanofibres as exemplified for $2 \% \mathrm{CNF}-\mathrm{YSZ}$ and $3 \% \mathrm{CNF}-\mathrm{Al}_{2} \mathrm{O}_{3}$ (Fig. 1 band Fig. S2A). These compositions were also used in the microstructural and mechanical characterization of the green and sintered nanocomposite ceramics since both compositions are in the regime of electrical percolation

(cf. Fig. S8Àand discussion below). Significant mechanical reinforcement brought about by the CNF could be clearly demonstrated since cold isostatic pressing (CIP) at $250 \mathrm{MPa}$ of these powders renders highly compacted green bodies that displayed very high flexural strength of 17 and $15 \mathrm{MPa}$ for $2 \% \mathrm{CNF}-\mathrm{YSZ}$ and $3 \% \mathrm{CNF}-\mathrm{Al}_{2} \mathrm{O}_{3}$, respectively (Table S1 These values are an order of magnitude larger as compared to dry-pressed green bodies containing polymeric binders ${ }^{23}$ and thus, these CNFï ceramic composites are suitable for green machining, which is an industrially important pre-sintering rapid prototype shaping technique (Fig. 1c). ${ }^{24}$ SPS sintering of the $2 \% \mathrm{CNF}-\mathrm{YSZ}$ and $3 \% \mathrm{CNF}-\mathrm{Al}_{2} \mathrm{O}_{3}$ green bodies at $1250{ }^{\circ} \mathrm{C}$ and at $1350{ }^{\circ} \mathrm{C}$, respectively, yielded black coloured, dense nanocomposite ceramics (Fig. 1d and Table S1ㅡㅅ). 

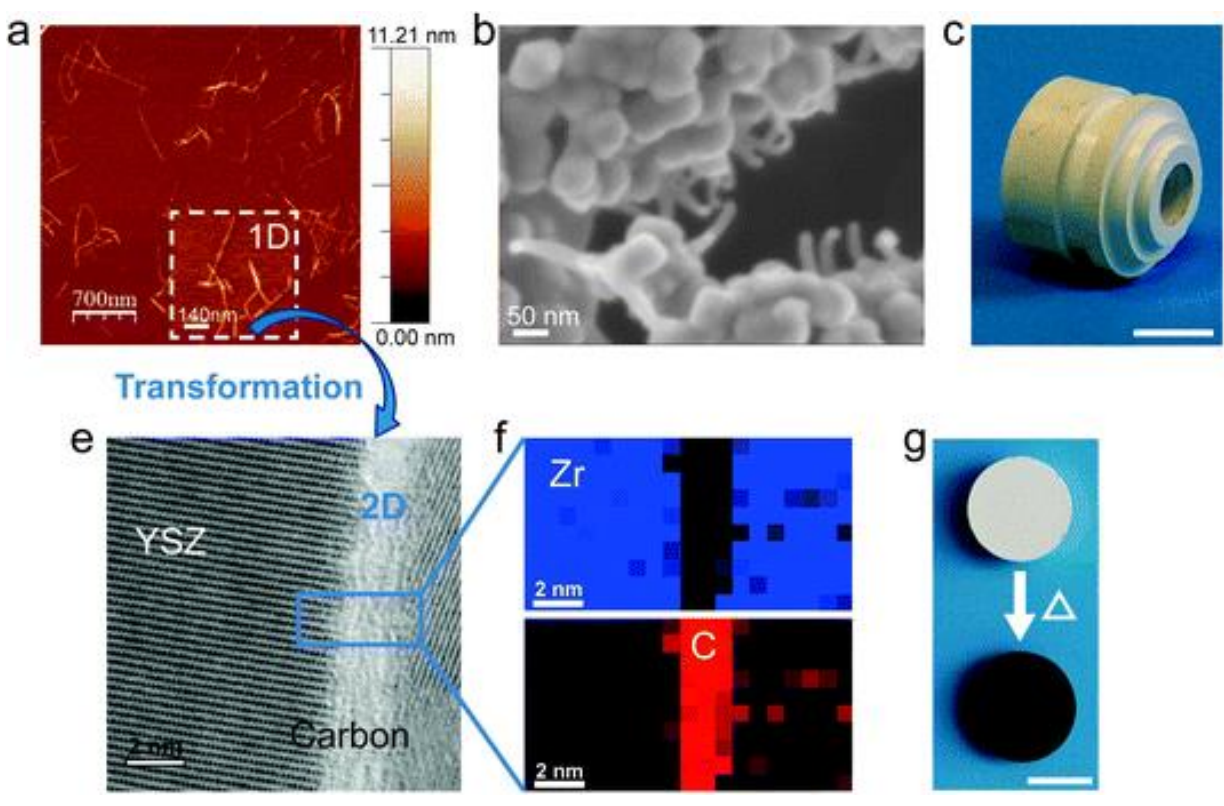

Fig. 1 Microstructure of green and SPS-sintered CNFï ceramics. (a) Atomic force microscopy (AFM) micrograph of individual cellulose nanofibres deposited on an APTES-modified mica substrate. The magnified inset image underlines the 1D morphology of CNF, which is transformed into a 2D carbon phase during SPS sintering as observable in e. (b) SEM micrograph of freeze-dried 2\%CNF-YSZ powder. (c) Photograph of a machined 2\%CNF-YSZ green body. (d) Photograph of 2\%CNF-YSZ before and after SPS sintering showing a white to black colour change. (e) BF STEM image of SPS sintered 2\%CNF-YSZ. (f) Electron energy loss spectroscopy (EELS) mapping across a grain boundary in 2\%CNF-YSZ using the carbon (C) and the zirconium $(\mathrm{Zr})$ signals, respectively. The scale bars in $\mathrm{c}$ and $\mathrm{d}$ refer to $1 \mathrm{~cm}$, respectively.

Energy dispersive X-ray spectroscopy (EDX) mapping (Fig. S3쓰) and bright and dark field STEM images (Fig. 2a) of the sintered composites revealed a continuous carbon phase, which is uniformly distributed within the material at the macroscopic and microscopic levels, respectively. At the nanoscale level, STEM imaging (Fig. 1e) and quantitative EELS mapping (Fig. 1f) demonstrate that the carbon phase is concentrated as an intergranular film forming a $2 \ddot{\mathrm{i}} 3 \mathrm{~nm}$ thick grain boundary (GB) layer completely surrounding the grains (Fig. 2b). These observations suggest that the 1-dimensional cellulose nanofibres are transformed into a 2-dimensional carbon film during SPS sintering ( $c f$. Fig. 1a and e). In fact, there is clear evidence from HRTEM analysis that this carbon film has a layered microstructure in both 
ceramic nanocomposites (Fig. S4À). Fig. 2c shows graphitic layers with an interlayer distance of $3.4 \AA$ matching the $c$-axis lattice constant of graphite or the distance between stacked graphene layers. $\frac{25}{-}$ The observed films consist of $6 і 10$ layers typical of few-layered graphene (FLG). ${ }^{26}$ Graphitic carbon was also confirmed by core level C 1s X-ray photoelectron spectroscopy (XPS) (Fig. S5À) and in Raman spectra (Fig. 2d). The latter are characterized by the $\mathrm{D}$ and $\mathrm{G}$ bands at 1350 and $1595 \mathrm{~cm}^{\overline{1} 1}$, respectively, as well as the 2D band at $2704 \mathrm{~cm}^{\mathrm{i} 1}$. While the $\mathrm{G}$ band is attributed to the in-plane $\mathrm{CïC}$ vibrations of $\mathrm{sp}^{2}$ hybridised carbon atoms, the $2 \mathrm{D}$ band originates from second-order phonon processes sensitive to the stacking order of graphene layers. $\frac{26}{}$
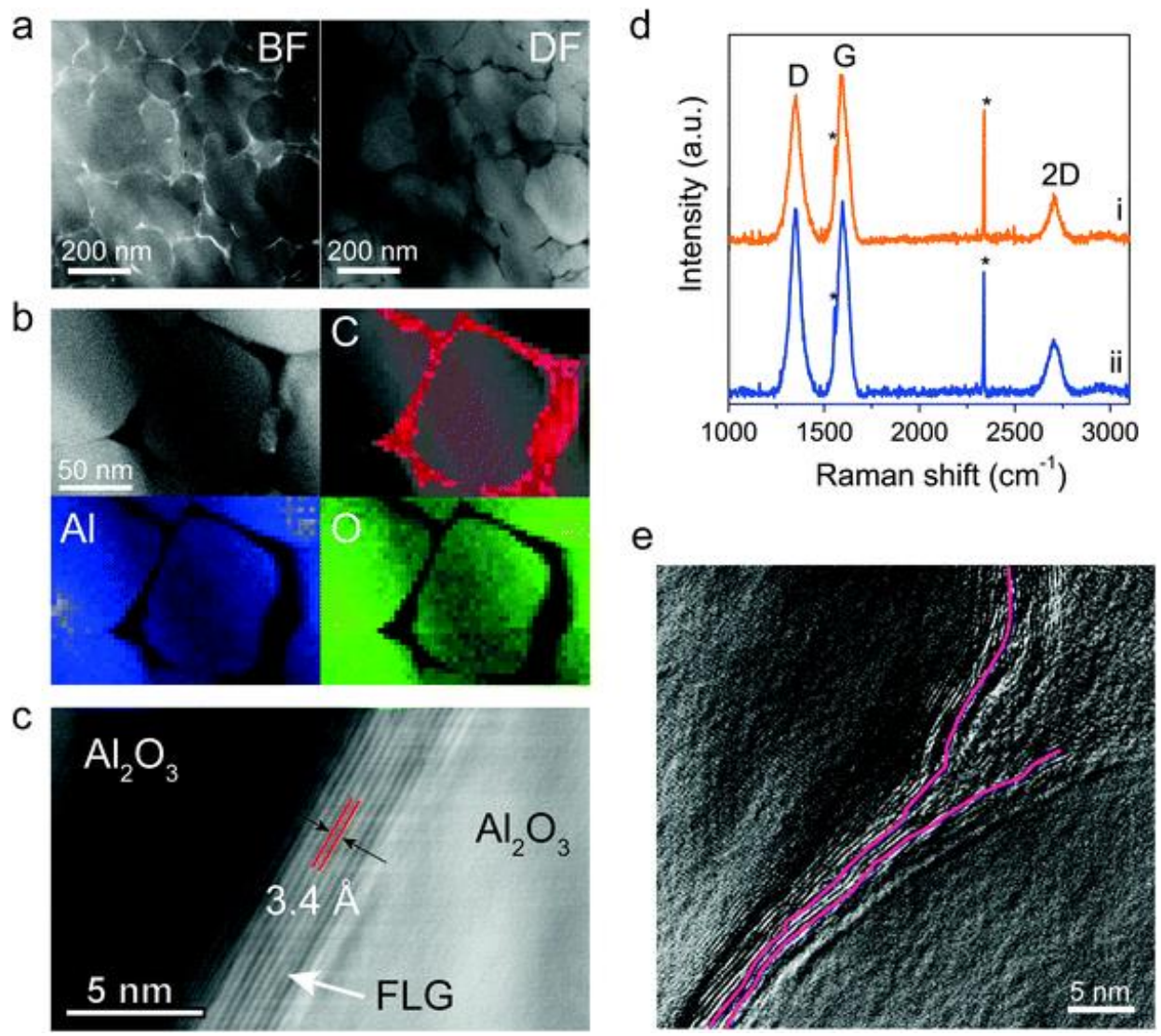

Fig. 2 Microstructure and formation of intergranular FLG films. (a) STEM bright field (BF) and dark field (DF) images of SPS sintered 2\%CNF-YSZ. The contrast at the ceramic grain boundaries (GBs) in the $\mathrm{BF}$ and $\mathrm{DF}$ mode is indicative of a continuous intergranular carbon network. (b) DF STEM image of $\mathrm{Al}_{2} \mathrm{O}_{3}$ grains and the surrounding GB phase in $3 \% \mathrm{CNF}-\mathrm{Al}_{2} \mathrm{O}_{3}$. Color EELS maps that use the signals of the $\mathrm{C} \mathrm{K}_{1}$ edge, $\mathrm{Al}$ $\mathrm{L}_{2,3}$ edge, and $\mathrm{O} \mathrm{K}_{1}$ edge, respectively, show that the GB consists of a carbon-containing phase, while $\mathrm{Al}$ and $\mathrm{O}$ are confined in the bulk of the grains. (c) BF STEM image of a few-layered graphene (FLG) film between $\mathrm{Al}_{2} \mathrm{O}_{3}$ grains in $3 \% \mathrm{CNF}-\mathrm{Al}_{2} \mathrm{O}_{3}$. An atomic 
plane distance of $3.4 \AA$ is indicated. (d) Raman spectra of SPS sintered 2\%CNF-YSZ (i) and $3 \% \mathrm{CNF}-\mathrm{Al}_{2} \mathrm{O}_{3}$ (ii). ${ }^{*}=\mathrm{O}_{2}(\mathrm{~g})$ and $\mathrm{N}_{2}(\mathrm{~g})$. (e) TEM image of SPS sintered $3 \% \mathrm{CNF}-$ $\mathrm{Al}_{2} \mathrm{O}_{3}$ highlighting a $\mathrm{Y}$ junction of the intergranular FLG films that fuse together.

The unimodal shape and width $\left(\mathrm{FWHM}=70 \mathrm{~cm}^{i 1}\right)$ of the recorded band suggest a multilayered graphene phase, which is in agreement with the HRTEM observations. Nonetheless, the presence of a pronounced $\mathrm{D}$ band (intensity ratio $I_{\mathrm{D}} / I_{\mathrm{G}}$ å 0.9 ) in the spectra also indicates that the FLG contains defects possibly arising from graphitic crystal edges, point defects, and $\mathrm{sp}^{3}$ hybridized carbon atoms. ${ }^{26}$ It should be noted, however, that cellulose is usually considered as a non-graphitisable carbon source, but cellulose of high crystallinity and purity like cellulose nanofibres may indeed show capability for graphitisation. ${ }^{27}$ The cellulose nanofibres used in this work displayed a crystallinity index of $61 \%$ and a crystallite size of $1.9 \mathrm{~nm}$ typical of CNF from eucalyptus. $\frac{28}{-}$ Interestingly, the distance between the glucopyranose planes in cellulose $I_{6}$ is $3.8 \AA$ ( $0.5 a$ of the cellulose $I_{6}$ unit cell), ${ }^{29}$ which is close to the interlayer distance of graphite (i.e. $3.35 \AA$ ). This circumstance possibly favours the generation of high quality graphitic multilayers from crystalline cellulose $\mathrm{e}^{30}$ even though a full understanding of the exact mechanism is still challenging. ${ }^{27}$ Nevertheless, supporting this assumption, we could demonstrate a clear effect of CNF versus glucose on the electrical properties of sintered YSZ nanocomposites. With dissolved glucose as the carbon source the resulting material displayed a 75\% reduced electrical conductivity (vide infra) when compared to the equivalent $2 \% \mathrm{CNF}-\mathrm{YSZ}$ material. This result suggests that the intrinsic crystalline arrangement of the glucopyranose units in CNF as opposed to the randomly distributed glucose molecules indeed seems to favour the formation of a percolating network. A schematic illustration of the conversion of the CNFï powder nanocomposite into the FLGï ceramic nanocomposite during SPS sintering is presented in Fig. 3 also showing the lattices of cellulose $I_{6}$ and graphene. 


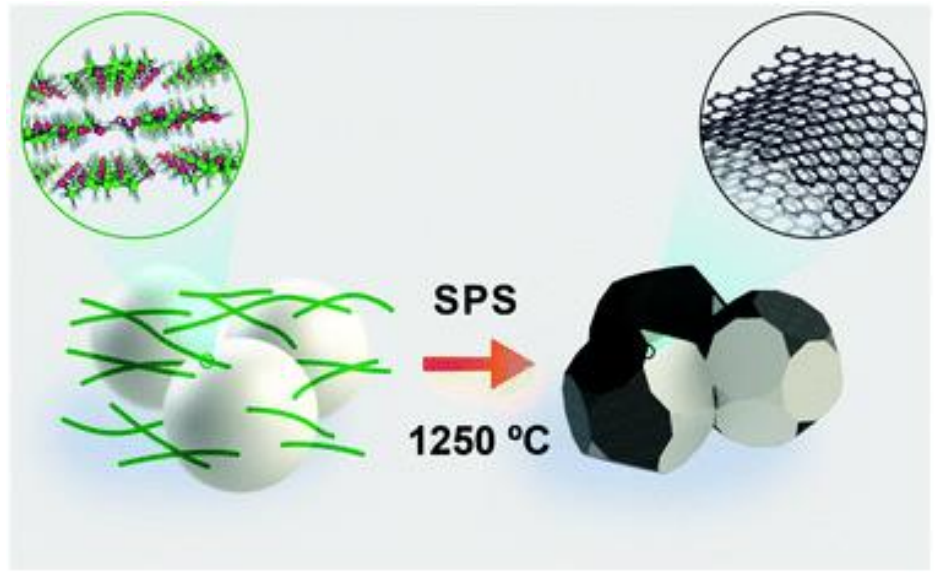

Fig. 3 Schematic illustration of the conversion of the CNFï powder nanocomposite into a FLGï ceramic nanocomposite. The lattices of cellulose $I_{6}$ and graphene are included

Interestingly, the FLG films follow the contour of the grains (Fig. 2e and S4aA) suggesting a high ability of bending and wrapping similar to individual graphene sheets. ${ }^{3.31}$ This specific behaviour and the resultant morphology is inherited from the flexible cellulose nanofibres that wrapped around the grains in the nanocomposite green body ( $c f$.Fig. 1b). During SPS sintering the CNF network transformed into thin graphene-like sheets maintaining the 3D topology and connectivity of the original network (Fig. 2e). This process evokes certain similarities to the high temperature covalent fusion of carbon nanotubes ${ }^{32}$ or graphene sheets ${ }^{23}$ forming interconnected 3D networks. ${ }^{4}$ Indeed, the high electrical conductivity ( $c f$. Fig. S8À) of the FLG-ceramics suggests that the cellulose-derived FLG sheets are bonded together reaching the percolation threshold. A ñYòjunction of three FLG films can be observed in Fig. 2e. On the other hand, the high defect concentration inferred from the intense Raman D band could be associated with vacancies and interstitials generated at the junction of the FLG sheets. ${ }^{3233}$ Also, the finite size effects in FLG due to their confinement between grains in the ceramic matrix as well as a lack of long ranged crystallinity coherence of the grown FLG cannot be ruled out as the cause of the large intensity of the D peak..$^{34}$

An estimation of the carbon content in the ceramics from the XPS spectra (Fig. S6À) yielded values below $1 \mathrm{wt} \%$, which was corroborated by elemental analysis. However, these measurements were ambiguous due to the low carbon content and possible carbon readsorption during the XPS analysis. It is also important to note that the carbon diffused neither into the $\mathrm{Al}_{2} \mathrm{O}_{3}$ nor the YSZ grains, as observed in the corresponding EELS maps (Fig. 
$\underline{2 \mathrm{~b}}$ and S7,Àrespectively). For instance, the quantification of the GB layer composition in $2 \% \mathrm{CNF}-\mathrm{YSZ}$ suggests that $87 \%$ is carbon and $13 \%$ is zirconium, while oxygen is not encountered in this layer (Fig. S7À).

The graphene-like carbon acts as an electro-conductive nanofiller and both alumina- and YSZ-based composites show extremely increased electrical conductivity in the range of 400 $\mathrm{S} \mathrm{m}{ }^{i 1}$ for $4 \% \mathrm{CNF}-\mathrm{Al}_{2} \mathrm{O}_{3}$ and $250 \mathrm{~S} \mathrm{~m}^{i 1}$ for $3 \% \mathrm{CNF}-\mathrm{YSZ}$. The percolation threshold for carbon-based electrical conductivity is as low as 1 and $0.5 \mathrm{wt} \% \mathrm{CNF}$, respectively, (Fig. S8À), which is on par or even below the typical values obtained for graphene or carbon nanotube reinforced ceramics. $\frac{1135}{}$ Our results are significant because the conductivity values encountered would usually require considerably higher amounts of nanofiller (1ï 10\%), which, however, negatively affect the microstructural homogeneity and downgrades the mechanical properties.

Fig. 4 displays the significant changes in the dielectric and charge transport properties of the sintered composites. The conductivity $(\sigma \mathrm{N}) \mathrm{j} v s$. frequency $(f)$ data for $x \mathrm{CNF}-\mathrm{YSZ}$ are shown in Fig. 4a together with the equivalent circuit fits. The values extracted from the fits at different temperatures $(T \mathrm{~s})$ were plotted on the Arrhenius axes (Fig. 4b), which shows the pronounced transition from the well-known ionic oxygen vacancy conduction in pure $\mathrm{YSZ}^{36}$ to mixed ionic/electronic conduction (MIEC) in 0.5\%CNF-YSZ with dominating ionic contributions. The signs of ionic conduction are also clearly visible in the complex impedance plots of i Z ZNjjs. ZNijig. 4c) and in the dielectric permittivity $\varepsilon N j s . f$ (Fig. 4c inset). In the former, the low- $f$ interface (IF) pike is a hallmark feature of ionic conductivity, whereas in the latter the massive linear increase in dielectric permittivity at the low $f$-end is associated with ionic conductivity too..$^{37}$ The GB and bulk activation energies for the ionic contributions were similar in the 0\%CNF-YSZ and 0.5\%CNF-YSZ samples $(0.9111 .0 \mathrm{eV})$, and the absolute conductivity values were unaffected by the CNF in the grain interior bulk regions. The latter is a clear confirmation of the findings from compositional analyses that the $\mathrm{CNF}$ derived carbon does not diffuse into bulk areas. On the other hand, the ionic conductivity in the GB areas is lower in the presence of $\mathrm{CNF}$, because graphitised GB layers may block some ionic pathways. Although the ionic conductivity is dominant in $0.5 \% \mathrm{CNF}$ YSZ, the electronic contribution is visible at high $f$ in the plots of $\sigma \mathrm{Njs}$. $f$ by an unusual upturn (Fig. 4a). The electronic contributions become dominating at CNF concentrations of Ó wt\%. MIEC is indicated in the presence of CNF, and the composites of higher and percolating CNF concentrations may be highly relevant for application as an electrode material in SOFCs. 
a

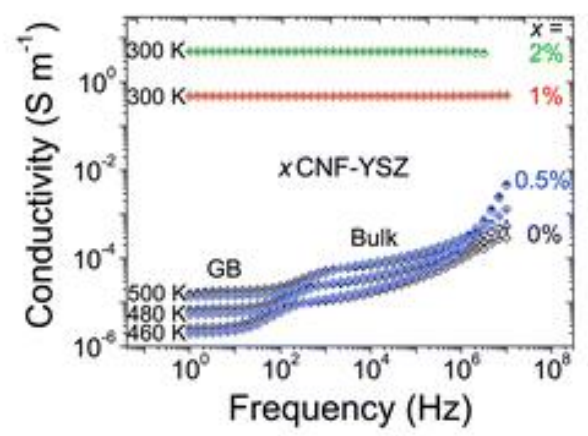

C

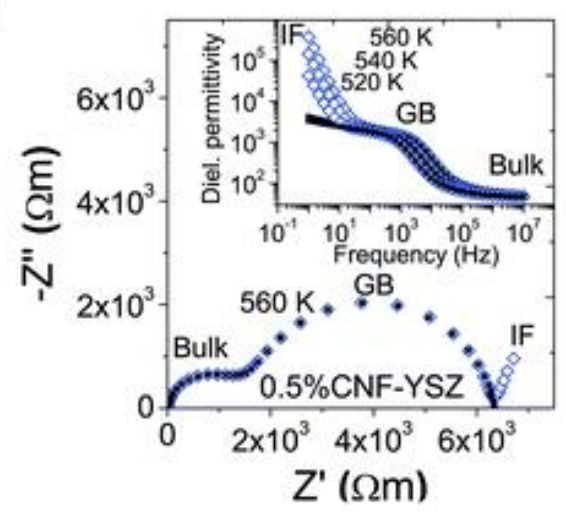

b

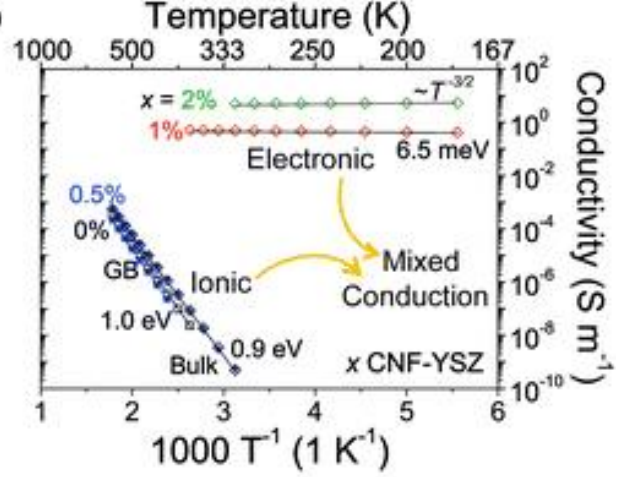

d

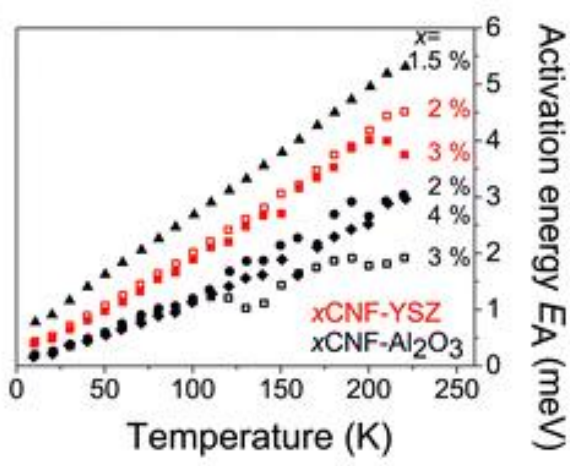

e

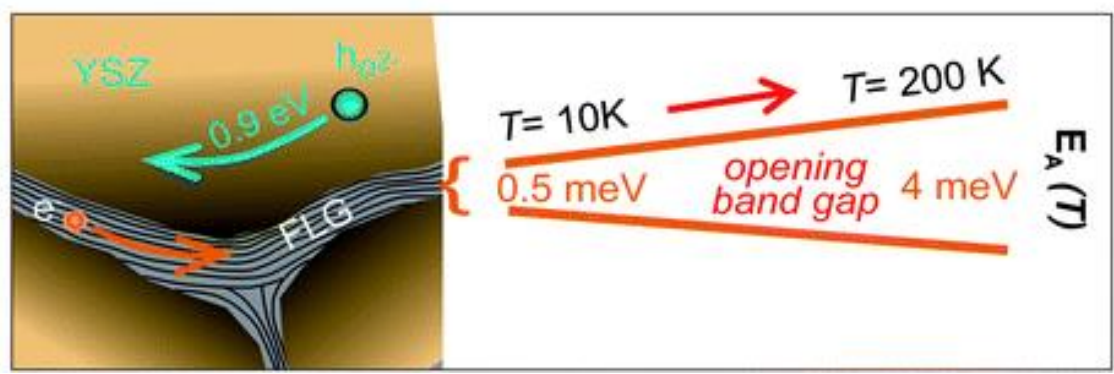

Fig. 4 Transport properties of the sintered nanocomposite ceramics: impedance spectroscopy data (open symbols) were fitted to a standard equivalent circuit model (black solid squares) containing 1 or 2 standard RQ elements of an ideal resistor $(R)$ and parallel non-ideal capacitor $(Q)$. The RQ elements were connected in series to represent GB and bulk contributions. ${ }^{37}$ (a) Conductivity $\sigma \mathrm{Nj} s$. frequency $f$ plots of $x \mathrm{CNF}-\mathrm{YSZ}$. The $0.5 \%$ CNF-YSZ curve shows a high- $f$ upturn, modelled by an additional series resistor. (b) Plots of $\sigma \mathrm{Njs}$. 1000/T plots for $x \mathrm{CNF}-\mathrm{YSZ}$ showing good linearity. The $\sigma$ Njvalues were obtained from circuit fits, and the activation energies for GB (solid symbols) and bulk (open symbols) conduction were derived from the corresponding Arrhenius plots. 
The 0\%CNF-YSZ (pure YSZ) is a purely ionic conductor, the 0.5\%CNF-YSZ is an MIEC with dominating ionic, and the 1\%CNF-YSZ is an MIEC with dominating electronic conductions. (c) Complex impedance plot ī ZMNjs. ZNjand dielectric permittivity $\varepsilon \mathrm{Njs}$. $f$ (inset) of $0.5 \% \mathrm{CNF}-\mathrm{YSZ}$. The open symbols correspond to experimental data and full squares correspond to fits to the equivalent circuit model. (d) Activation energies $E_{\mathrm{A}}(T) v s . T$ for $x \mathrm{CNF}-\mathrm{YSZ}$ and $x \mathrm{CNF}-\mathrm{Al}_{2} \mathrm{O}_{3} . E_{\mathrm{A}}(T)$ in the FLG grain boundary phase is $T$-dependent increasing from 0.5 to higher values in the range of 2 ï 6 meV. (e) Schematic illustration of the FLG films at a triple junction between the ceramic grains. Here the case of YSZ is demonstrated; the equivalent features can be observed at the triple grain junctions in sintered $\mathrm{CNF}-\mathrm{Al}_{2} \mathrm{O}_{3}$ nanocomposites. The electron $\left(\mathrm{e}^{\mathrm{i}}\right)$ and ionic oxygen vacancy $\left(h_{\mathrm{O}^{2 i}}\right)$ conduction mechanisms of YSZ are indicated. The activation energy, $E_{\mathrm{A}}(T)$, for oxygen vacancy conduction in the YSZ bulk is $0.9 \mathrm{eV}$, while the $E_{\mathrm{A}}(T)$ of electron conduction in the FLG grain boundary phase is $T$ dependent.

It should be further noted that the sintered $1 \% \mathrm{CNF}-Y$ SZ sample showed a semiconducting $T$-dependence of conductivity with a small charge transport gap of å6.5 meV (Fig. 4b), whereas the 2\%CNF-YSZ sample shows a metallic å $T^{3 / 2}$ dependence, which, however, was shown to be extrinsic. The correct dc resistance was therefore determined by the 4-point van-der-Pauw measurements at various $T$. The resulting Arrhenius curves (Fig. S9À) show a certain degree of non-linearity, which indicates a $T$-dependent activation energy $E_{\mathrm{A}}(T)$ (Fig. 4d) due to an opening charge transport gap upon heating (Fig. 4e). The $E_{\mathrm{A}} v s$. $T$ curves for sintered $x \mathrm{CNF}-\mathrm{YSZ}$ and $x \mathrm{CNF}-\mathrm{Al}_{2} \mathrm{O}_{3}$ in Fig. $4 \mathrm{~d}$ are qualitatively similar indicating an opening gap as part of the equivalent charge transport mechanism, which therefore may well originate from the carbon layers instead of the very different charge transport in $\mathrm{YSZ}_{\text {or }} \mathrm{Al}_{2} \mathrm{O}_{3}$. The temperature dependence of this small transport gap in the graphene-like 2D phase may originate from the crumpling of the carbon layers, ${ }^{31}$ in turn influenced by local stress fields resulting from a thermal expansion mismatch. The proposed ionic conduction and MIEC mechanisms in $x$ CNF-YSZ are illustrated in Fig. 4e. In the case of $x \mathrm{CNF}-\mathrm{Al}_{2} \mathrm{O}_{3}$ a transition from an insulating $\mathrm{Al}_{2} \mathrm{O}_{3}$-type charge transport to semiconducting graphene-like conduction was observed ( $c f$. Fig. S9Ầ). Alternatively, variable-range hopping (VRH) models have been applied previously to explain non-linear Arrhenius plots. ${ }^{38}$ However, this approach may not be reasonable in systems with metallic and 
delocalized or weakly localised electrons with a small gap since VRH is a localised electron hopping model based on fully localised electron tunnelling exhibiting larger transport gaps. ${ }^{39}$

Another remarkable property of the CNF-YSZ ceramics is demonstrated by the plot of the electrical conductivity together with the thermal conductivity at $300 \mathrm{~K}$ vs. the CNF doping level (Fig. 5a). The electrical conductivity increases by 14 orders of magnitude, while the thermal conductivity increases only by $6 \%$, comparing YSZ and $2 \%$ CNF-YSZ. Usually, the thermal and electrical conductivities are strongly coupled due to the thermal contribution of electron conduction. ${ }^{40}$ A big challenge in applications like thermoelectrics is therefore the de-coupling of these two transport properties ${ }^{103841}$ as demonstrated recently in metallic vanadium dioxide nanowires. ${ }^{40}$ Here, we show electric and thermal de-coupling in macroscopic materials with immediate implications for the above-mentioned technological applications. The nanometric dimensions of the graphene network in the nanocomposite ceramics are likely to give rise to a high thermal interface or phonon resistance similar to carbon nanotube composites..$^{38}$ 


\section{a}
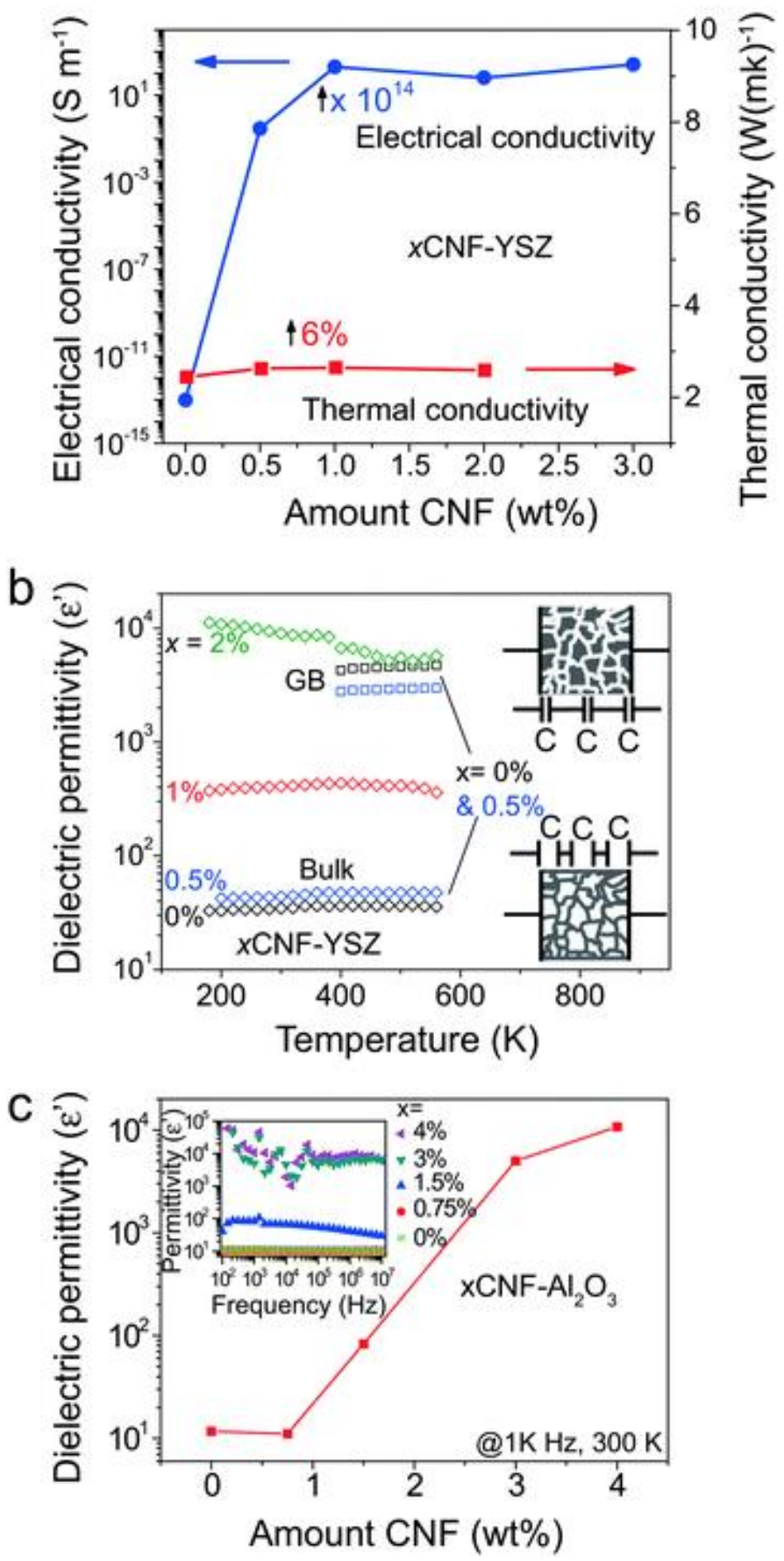

Fig. 5 Electronic and thermal conductivity, dielectric permittivity. (a) Electrical and thermal conductivity vs. CNF doping level in YSZ composites measured at $300 \mathrm{~K}$. (b) Dielectric permittivity $\varepsilon \mathrm{Nj} s$. T of sintered $x$ CNF-YSZ, where bulk and GB permittivity is indicated. The inset schemes indicate the dielectrically active areas (white colour) in terms of the ceramic grains for the bulk and the FLG layers for the GB contributions. Both contributions can be represented by thin (GB) or thick (bulk) capacitors connected 
in series. The $\varepsilon$ Njalues were obtained from the equivalent circuit fits of dielectric spectra. (c) $\varepsilon$ Njalues at $1 \mathrm{~K} \mathrm{~Hz}$ and $300 \mathrm{~K}$ of sintered $x \mathrm{CNF}-\mathrm{Al}_{2} \mathrm{O}_{3}$ as a function of the $\mathrm{CNF}$ content. The inset shows the $\varepsilon \mathrm{Nj}$ s. frequency.

Furthermore, the sintered CNF-ceramics showed striking improvements in the dielectric permittivity, which is relevant for miniaturising communication devices. ${ }^{6.42}$ High dielectric permittivity \&Njalues of up to $10^{4}$ were detected with increasing $\mathrm{CNF}$, which represents giant increases of $250 \times$ and $1000 \times$ for $x \mathrm{CNF}-\mathrm{YSZ}$ and $x \mathrm{CNF}-\mathrm{Al}_{2} \mathrm{O}_{3}$, respectively (Fig. 5b and c), similar to the $\varepsilon \mathrm{Nj}$ values reported for graphene $/ \mathrm{Al}_{2} \mathrm{O}_{3}$ composites. ${ }^{6} \mathrm{Such}$ a massive enhancement is not likely to be an intrinsic ceramic effect and, therefore, the alternative explanation of the formation of microscopic capacitors between the ceramic grains at the FLG GB areas may be favourable (Fig. 5b insets). .453

In addition to the reported improvements in the electrical and dielectric properties, the incorporation of CNF also improved the mechanical properties of both the dry-pressed green body (vide supra) and the sintered samples. For instance, the Vickers hardness $H_{\mathrm{v}}$ of the sintered 2\%CNF-YSZ and 3\%CNF- $\mathrm{Al}_{2} \mathrm{O}_{3}$ was 14.2 and $21.2 \mathrm{GPa}$, respectively, which is on par with pristine YSZ and several GPa higher than for pure coarse-grained alumina. On the other hand, the indentation toughness was 4.4 and 4.0 MPa m ${ }^{0.5}$ (2\%CNF-YSZ and 3\%CNF$\mathrm{Al}_{2} \mathrm{O}_{3}$, respectively) (Table $\mathrm{S} 1 \underline{A}$ ), which is on par or slightly higher than the values of commercial benchmark ceramics and also nanocarbon reinforced composite ceramics. ${ }^{\frac{7.144}{144}}$ However, it has to be emphasised with respect to the latter, that especially grapheneï ceramic nanocomposites are prone to the directional anisotropy of electrical versus mechanical properties as a result of the alignment of graphene flakes and hence, hampering the applicability of such materials, ${ }^{45}$ which is not the case for the present CNFï ceramics system. The good mechanical properties of the sintered CNFï ceramics can be related to a microstructural refinement, where the FLG phase could successfully prevent the excessive grain growth during sintering by pinning the grain boundaries (Fig. S10 and S11À). An additional contribution stems from a toughening effect conveyed by the carbon phase. This effect was suggested from the fractographic analysis that demonstrated crack branching, bridging, and deflection (Fig. 6a). Occasionally, also carbonaceous layers were debonded from the ceramic matrix that bridged the crack (Fig. 6b). Both observations are common for fibre or sheet reinforced ceramics ${ }^{11.446}$ and can contribute to further increase the toughness as a result of reduced stress intensity at the crack tip. Therefore, it can also be assumed that the ex-cellulose FLG behaves like a sheet or fibre reinforcement. It is well- 
known from natural biomineralised nanocomposite materials like nacre or bone that toughness is enhanced in a brittle material through the presence of nanometre thick and soft carbon-rich interfaces..$^{47}$
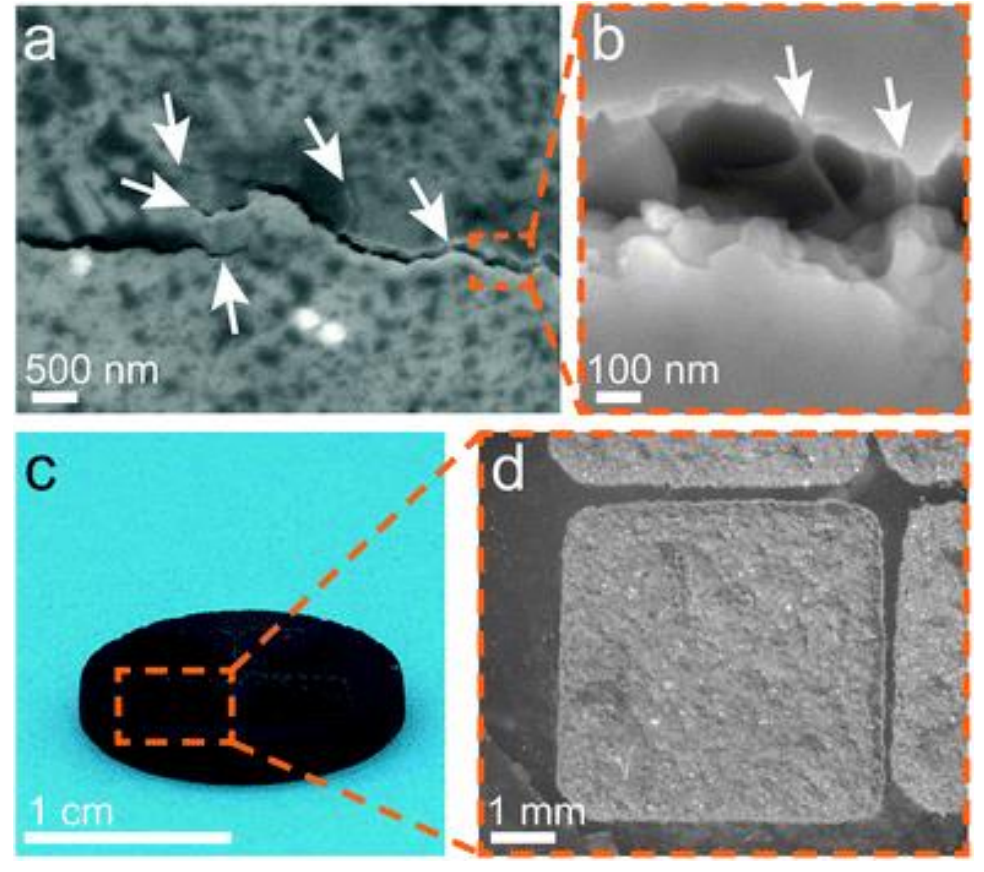

Fig. 6 Fracture behaviour and EDM application. (a) SEM micrograph of an arrested crack in sintered 2\%CNF-YSZ after the Vickers indentation showing crack branching, bridging and deflection (white arrows) induced by the carbonaceous phase. (b) A closeup of (a) showing crack bridging of the FLG sheets debonded from the ceramic matrix. (c) Photograph of sintered 3\% CNF- $\mathrm{Al}_{2} \mathrm{O}_{3}$ showing electro-discharge erosion pits and, (d) a close up of (c) made with a stereomicroscope.

Finally, the sintered CNFï ceramics were tested for electro-discharge machining (EDM) as a technologically relevant subtractive manufacturing method for the complex shaping of dense and hard (sintered) materials. Fig. $6 \mathrm{c}$ and d show the erosion pits on the surface of $3 \% \mathrm{CNF}-\mathrm{Al}_{2} \mathrm{O}_{3}$ produced by die-sinking $\mathrm{EDM}$. The volumetric material removal rate (MRR) was $0.33 \mathrm{~mm}^{3} \mathrm{~min}^{\overline{1} 1}$ and the die-sink rate was $12.4 \mathrm{\varepsilon m} \min ^{i 1}$. Even though this MRR was about $50 \%$ lower at the given pulse duration and current as compared to using conventional $\mathrm{TiN}$ or TiC fillers ${ }^{48}$ it should be remarked that these higher MRRs were typically achieved at approximately $30 \ddot{1} 40$ vol\% filler content. In addition, the MRR should also be grain-size dependent, whereas in our case finely grain-sized samples will result in a slower EDM process. On the whole these results show that CNF incorporation represents a versatile 
alternative to fabricate EDM suitable materials with a considerably lower second phase content (1 wt\% vs.50 wt\%) as compared to conventional EDM materials ${ }^{49}$ and are competitive with multiwall carbon nanotubes as EDM fillers..$^{50}$

\section{Conclusions}

In summary, we report the use of natural nanofibres for the in situ generation of 3D percolating graphene-like networks embedded in engineering ceramics. The transformation of the cellulose nanofibres into a continuous, graphene-based architecture during sintering is the key to the remarkable electronic and dielectric properties of the microstructurally refined FLGï ceramic composites, while at the same time the thermal conductivity is decoupled. Besides the transport properties, the incorporated cellulose nanofibres largely improve the mechanical properties and also enable the use of technologically important machining methods for electro-conductive ceramics. We envisage that our results can advance the processing science and technology to provide the improved hierarchical graphene composite materials needed for advanced applications in fields like energy and telecommunications.

\section{Experimental}

\section{Materials}

In the present work, commercially available $\mathrm{U}_{-} \mathrm{Al}_{2} \mathrm{O}_{3}$ (Taimicron TM-DAR, Taimei Chemicals Co., Ltd, Japan) and 3 mol\% $\mathrm{Y}_{2} \mathrm{O}_{3}$-stabilized $\mathrm{ZrO}_{2}$ (YSZ; TZ-3Y-E, Tosoh, Japan) powders were used. Cellulose nanofibres, extracted from never-dried eucalyptus pulp, were provided by Prof. Mutje, University of Girona, Spain. In brief, an aqueous suspension of the pulp was subjected to a TEMPO (2,2,6,6-tetramethylpiperidine 1-oxyl)-mediated oxidation step to render carboxylated fibres (1.39 $\mathrm{mmol} \mathrm{g}^{\top 1}$ charge). The subsequent mechanical disintegration using a high-pressure homogenizer (Panda + 2000, GEA, Italy) renders fully defibrillated cellulose nanofibres. The mechanical treatment results in a highly viscous $\mathrm{CNF}$ dispersion with a concentration of about $1 \mathrm{wt} \%$ and a $\mathrm{pH}$ of 7 . All other chemicals were purchased from Sigma-Aldrich.

\section{Powder processing and sintering}


Nanocomposite powder. Aqueous powder slurries (30 vol\% solid loading) were prepared

from citric-acid-stabilised $\breve{\mathrm{U}} \mathrm{Al}_{2} \mathrm{O}_{3}$ and YSZ nanoparticles, respectively, and adjusted to $\mathrm{pH}$ 8.5. The slurries were mixed with aqueous $\mathrm{CNF}$ gel to render $\mathrm{CNF}$ : nanoparticle ratios of $0.5 \mathrm{I} 4 \mathrm{wt} \%$ (1.85ï 10 vol\%). The mixtures were attrition milled (3-millimetre-sized $\mathrm{ZrO}_{2}$ milling balls) for $3 \mathrm{~h}$ following rapid freezing in liquid nitrogen and a freeze-drying step to obtain dry nanocomposite $\mathrm{Al}_{2} \mathrm{O}_{3}$ and $\mathrm{YSZ}$ powder. For comparison, a nanocomposite of YSZ containing $2 \mathrm{wt} \%$ of glucose was prepared by adding the corresponding amount of aqueous glucose solution to the YSZ powder slurry.

Green bodies. For the evaluation of the $\mathrm{CNF}$ reinforcement effect on green bodies the nanocomposite powders were cold isostatically pressed with $250 \mathrm{MPa}$. The green bodies were evaluated by green machining and by measuring flexural strength using a piston-onthree-balls setup.

Spark plasma sintering (SPS). For SPS sintering, $2 \mathrm{~g}$ of the nanocomposite powder was added into a $16 \mathrm{~mm}$ or $20 \mathrm{~mm}$ diameter graphite sintering die containing $0.5 \mathrm{~mm}$ thick graphite paper. The die was isolated with $5 \mathrm{~mm}$ thick graphite wool. The sintering was performed in an SPS furnace (SPS-615E, Dr SINTER; SPS Syntex Inc., Japan) under vacuum conditions. The heating rate was set to $100{ }^{\circ} \mathrm{C} \min ^{i 1}$, while the initial contact pressure was increased from $3 \mathrm{kN}$ to a final pressure corresponding to $80 \mathrm{MPa}$ in 1 minute during the most pronounced densification of the sample. The nanocomposite consolidated powders were sintered at a final temperature of $1250{ }^{\circ} \mathrm{C}$ (YSZ containing samples) and at $1350{ }^{\circ} \mathrm{C}\left(\mathrm{Al}_{2} \mathrm{O}_{3}\right.$ containing samples $)$ with 5 minutes dwell time.

\section{Characterization}

Density. The densities of the SPS-sintered samples were determined using the Archimedes method, where deionized water was used as the immersion medium.

Microstructural analysis. The microstructure of the samples was characterised by fieldemission scanning electron microscopy (FE-SEM; JEOL JSM 7600F) and focused ion beam SEM (FIB-SEM; Helios Nanolab 650, FEI, Hillsboro, USA). The average grain size was evaluated by measuring at least 100 grains from the SEM images of the fractured surfaces using the linear intercept method. No correction factor was used. The X-ray diffraction (XRD) patterns of sintered specimens were recorded using a PANalytical X'pert PRO MPD 
diffractometer (PANalytical, Almelo, the Netherlands) equipped with a PIXcel detector and using a $\mathrm{Cu}-\mathrm{K}_{\breve{u}}$ radiation source. The measurements were carried out using fixed divergence $\left(1^{\circ}\right)$ and anti-scattering slits, a $10 \mathrm{~mm}$ mask, and a continuous scan mode in the $2 \theta$ range $20 \ddot{\mathrm{i}}$ $100^{\circ}$. Transmission electron microscopy characterisation was performed on a scanning transmission electron microscope with atomic resolution (Jeol STEM ARM 200 CF) in the TEM and dark/bright-field scanning transmission electron microscopy (DF/BF STEM) modes. The particle size distribution of the nanocomposite powders was assessed with a laser particle-size analyser (LA-920, Horiba, Japan). Atomic force microscopy (AFM) of individual cellulose nanofibres deposited on the APTES-modified mica substrate was performed with a Cervantes instrument from Nanotec electrónica S.L. (Spain) equipped with Nanosensors PPP-FMR cantilevers with a spring constant of $1.5 \mathrm{~N} \mathrm{~m}^{\overline{1} 1}$ and a resonance frequency of $75 \mathrm{kHz}$. Images were obtained under ambient conditions in a modulated amplitude mode.

Chemical analysis. The distribution of the carbon phase in the sintered ceramics was characterized by energy-dispersive X-ray spectroscopy (EDX) coupled to a (FEI NOVA Nano SEM 230) FE-SEM and by electron energy loss spectroscopy (EELS). The EDX maps were recorded with a Type SDD Apollo 10 EDAX detector and the data acquisition conditions were kept constant at a working distance of $6.5 \mathrm{~mm}$, a spot size of 5.5, a landing energy of $5.0 \mathrm{keV}$, and an accumulation time of $500 \mathrm{~s}$. The EELS spectra were collected in the STEM mode in scanned areas across the grain boundary; 24 mrad convergence and 60 mrad collection semi-angles were used. Low and core loss were acquired simultaneously using the Dual-EELS option of the system. The sample thickness where the EELS spectra were collected was between 30 and $50 \mathrm{~nm}$. The chemical composition and the state of the carbon atoms were analysed by X-ray photoelectron spectroscopy (XPS) on a PHI-TFA XPS spectrometer (Physical Electronics), by elemental analysis (CHNS PerkinElmer 2400) and by Raman spectroscopy (EZ-RAMAN-N series, Enwave Optronics Inc., laser excitation was $\lambda=532 \mathrm{~nm})$.

Electrical properties. The dielectric properties of the sintered composites were measured by alternating current (AC) impedance spectroscopy using a Novocontrol Alpha Analyser. Measurements were performed between $f=1 \mathrm{Hzï} 10 \mathrm{MHz}$ with a $0.1 \mathrm{~V}$ applied AC voltage signal, over the $T$-range of $160 \mathrm{Kï} 560 \mathrm{~K}$ (i $113{ }^{\circ} \mathrm{C}$ to $287^{\circ} \mathrm{C}$ ) upon heating cycles in $20 \mathrm{~K}$ temperature increments. The data were taken under steady-state conditions after the selected $T$ was stabilized for at least 10 minutes. Resistance as a function of magnetic field and temperature $(2 \ddot{\mathrm{i}} 300 \mathrm{~K})$ was measured on $8 \times 8 \mathrm{~mm}^{2}$ square samples using the van der 
Pauw configuration in a $9 \mathrm{~T}$ Quantum Design PPMS. Resistance measurements were taken in three configurations: two of them optimized for resistivity measurements along nominally orthogonal directions in the sample while the remaining configuration, with the current along the diagonal, maximized the Hall resistance.

Mechanical analysis. The biaxial flexural strength of the green and the SPS-sintered samples, where the latter were ground and polished on the tensile side (finished with $3 \mathrm{~cm}$ diamond paste), were measured with a piston-on-three-balls setup, according to the ISO 6872 standard, on a universal testing machine (Quasar 50; Galdabini, Italy) at a loading rate of $1 \mathrm{~mm} \min ^{i 1}$. The hardness and indentation toughness of the samples were determined using a Vickers indentation hardness tester (Zwick GmbH \& Co., Germany). The indents were made using a Vickers-type indenter by applying a 10 and $50 \mathrm{~N}$ load for $10 \mathrm{~s}$ for evaluating hardness and indentation toughness, respectively. The indentation toughness was then calculated directly from the crack lengths using the method of Niihara ${ }^{51}$ for Palmqvist cracks.

Electro-discharge machining. Electro-discharge machining (EDM) was performed on $2 \% \mathrm{CNF}-\mathrm{YSZ}$ and $3 \% \mathrm{CNF}-\mathrm{Al}_{2} \mathrm{O}_{3}$ samples by applying the die-sinking EDM principle on an IT Elektronika 200M-E machine (Slovenia). For the machining experiments, a rectangular $\left(5.17 \times 5.17 \mathrm{~mm}^{2}\right)$ copper electrode was used. The duration of the machining was set to 20 min using the following machining parameters: discharge current $i_{\mathrm{e}}=2 \mathrm{~A}$, ignition voltage $u_{\mathrm{i}}=280 \mathrm{~V}$, discharge duration $t_{\mathrm{e}}=17 \varepsilon \mathrm{s}$ and pulse interval $t_{\mathrm{o}}=36 \varepsilon \mathrm{s}$.

\section{Conflicts of interest}

There are no conflicts to declare.

\section{Acknowledgements}

Financial support from the Slovenian Research Agency (research core funding No. P2-0087) and the Spanish Ministry of Economy and Competitiveness (CYCIT, MAT2012-31759, MAT2015-71117-R, MAT2014-52405-C2-2-R) is acknowledged as well as from the EU Horizon 2020 research and innovation program under grant agreement No. 696656 (Graphene Core1-Graphene-based disruptive technologies). The authors thank Prof. Mutje and Dr Tarrés for providing CNF, D. Eterovil for photography, Dr I. Sabotin for EDM tests, A. Varela for EDX analysis, Dr Muñoz for access to a Raman microscope, Aljaǵ Ivekovil 
for assistance with illustrations, and Prof. Z. Shen for fruitful discussions. BW and JPG thank the Spanish MINECO for granting Juan de la Cierva fellowships (IJCI-2015-23886, FJCI-2015-24149).

\section{References}

1. N. T. Markov, M. Ercsey-Ravasz, D. C. Van Essen, K. Knoblauch, Z. Toroczkai and H. Kennedy, Science, 2013,342,1238406.

2. Z. Chen, W. Ren, L. Gao, B. Liu, S. Pei and H.-M. Cheng, Nat. Mater., 2011, 10, $424 i \ddot{i} 428$

3. A. Dasgupta, L. P. Rajukumar, C. Rotella, Y. Lei and M. Terrones, Nano Today, 2017, 12, $116 і ̈ 135$

4. K. Shehzad, Y. Xu, C. Gao and X. Duan, Chem. Soc. Rev., 2016, 45, 55411 5588

5. C. Cheng, S. Li, A. Thomas, N. A. Kotov and R. Haag, Chem. Rev., 2017, 117, $1826 і ̈ 1914$

6. K. Ahmad, W. Pan and H. Wu, RSC Adv., 2015, 5, 33607ї 33614

7. B. Yazdani, F. Xu, I. Ahmad, X. Hou, Y. Xia and Y. Zhu, Sci. Rep., 2015, 5, 11579

8. G.-D. Zhan, J. D. Kuntz, J. Wan and A. K. Mukherjee, Nat. Mater., 2003, 2, 3810 42

9. G. Pernot, M. Stoffel, I. Savic, F. Pezzoli, P. Chen, G. Savelli, A. Jacquot, J. Schumann, U. Denker, I. Mönch, C. Deneke, O. G. Schmidt, J. M. Rampnoux, S. Wang, M. Plissonnier, A. Rastelli, S. Dilhaire and N. Mingo, Nat. Mater., 2010, 9, $491 і ̈ 495$

10. F. J. DiSalvo, Science, 1999, 285, $703 і ̈ 706$

11. O. T. Picot, V. G. Rocha, C. Ferraro, N. Ni, E. D'Elia, S. Meille, J. Chevalier, T. Saunders, T. Peijs, M. J. Reece and E. Saiz, Nat. Commun., 2017, 8, 14425

12. M. Estili, A. Kawasaki and Y. Sakka, Adv. Mater., 2012, 24, 4322ï 4326

13. K. Markandan, J. K. Chin and M. T. T. Tan, J. Mater. Res., 2017, 32, 84ї 106

14. X. Wang, N. P. Padture and H. Tanaka, Nat. Mater., 2004, 3, 53910 544

15. O. Hanzel, J. Sedlál ek and P. Gajgalík, J. Eur. Ceram. Soc., 2014, 34, $18451 ̈ 1851$

16. S. Ling, D. L. Kaplan and M. J. Buehler, Nat. Rev. Mater., 2018, 3, 18016

17. B. Wicklein, A. Kocjan, G. Salazar-Alvarez, F. Carosio, G. Camino, M. Antonietti and L. Bergström, Nat. Nanotechnol., 2015,10, $277 \mathrm{ï} 283$

18. H. Zhu, W. Luo, P. N. Ciesielski, Z. Fang, J. Y. Zhu, G. Henriksson, M. E. Himmel and L. Hu, Chem. Rev., 2016, 116, $9305 і ̈ 9374$ 
19. A. Dufresne, Mater. Today, 2013, 16, 22010 227

20. R. J. Moon, A. Martini, J. Nairn, J. Simonsen and J. Youngblood, Chem. Soc. Rev., 2011, 40, 3941 3994

21. K. E. Shopsowitz, W. Y. Hamad and M. J. MacLachlan, Angew. Chem., Int. Ed., 2011, 50, $10991 і ̈ 10995$

22. Z. Li, J. Liu, K. Jiang and T. Thundat, Nano Energy, 2016, 25, 16111169

23. M. R. Ben Romdhane, T. Chartier, S. Baklouti, J. Bouaziz, C. Pagnoux and J. F. Baumard, J. Eur. Ceram. Soc., 2007, 27, $2687 і ̈ 2695$

24. S. Dhara and B. Su, Int. J. Appl. Ceram. Technol., 2005, 2, $262 і ̈ 270$

25. M. S. Dresselhaus, A. Jorio, M. Hofmann, G. Dresselhaus and R. Saito, Nano Lett., 2010, 10, $751 і ̈ 758$

26. A. C. Ferrari and D. M. Basko, Nat. Nanotechnol., 2013, 8, $235 \mathrm{Ï} 246$

27. A. G. Dumanlēand A. H. Windle, J. Mater. Sci., 2012, 47, $4236 i ̈ 4250$

28. Ú. Fillat, B. Wicklein, R. Martín-Sampedro, D. Ibarra, E. Ruiz-Hitzky, C. Valencia, A. Sarrión, E. Castro and M. E. Eugenio,Carbohydr. Polym., 2018, 179, $2521 \ddot{1} 261$

29. Y. Nishiyama, G. P. Johnson, A. D. French, V. T. Forsyth and P. Langan, Biomacromolecules, 2008, 9, 3133 ï 3140

30. D.-Y. Kim, Y. Nishiyama, M. Wada and S. Kuga, Carbon N. Y., 2001, 39, 1051 ï 1056

31. S. Deng and V. Berry, Mater. Today, 2016, 19, $197 і ̈ 212$

32. M. Terrones, F. Banhart, N. Grobert, J.-C. Charlier, H. Terrones and P. M. Ajayan, Phys. Rev. Lett., 2002, 89, 75505

33. J. Zhang, G. Shi, C. Jiang, S. Ju and D. Jiang, Small, 2015, 11, $6197 і ̈ 6204$

34. L. M. Malard, M. A. Pimenta, G. Dresselhaus and M. S. Dresselhaus, Phys. Rep., 2009, 473, 51 ï 87

35. P. Hvizdos, V. Puchy, A. Duszova, J. Dusza and C. Balazsi, Ceram. Int., 2012, 38, $5669 \ddot{1} 5676$

36. M. A. Frechero, M. Rocci, G. Sánchez-Santolino, A. Kumar, J. Salafranca, R. Schmidt, M. R. Díaz-Guillén, O. J. Durá, A. Rivera-Calzada, R. Mishra, S. Jesse, S. T. Pantelides, S. V. Kalinin, M. Varela, S. J. Pennycook, J. Santamaria and C. Leon, Sci. Rep., 2015, 5, 17229

37. R. Schmidt, in CRC Concise Encyclopaedia of Nanotechnology, ed. B. I. Kharisov, O. V. Kharissova and U. Ortiz-Mendez, CRC Press Taylor \& Francis Group, Boca Raton, USA, 2015, pp. $364 і ̈ 381$ 
38. W. H. Nam, Y. S. Lim, W. Kim, H. K. Seo, K. S. Dae, S. Lee, W.-S. Seo and J. Y. Lee, Nanoscale, 2017, 9, $7830 і ̈ 7838$

39. R. Schmidt, A. Basu and A. W. Brinkman, Phys. Rev. B: Condens. Matter, 2005, 72, 115101

40. S. Lee, K. Hippalgaonkar, F. Yang, J. Hong, C. Ko, J. Suh, K. Liu, K. Wang, J. J. Urban, X. Zhang, C. Dames, S. A. Hartnoll, O. Delaire and J. Wu, Science, 2017, 355, 371 ï 374

41. J.-K. Yu, S. Mitrovic, D. Tham, J. Varghese and J. R. Heath, Nat. Nanotechnol, 2010, 5, $718 і ̈ 721$

42. Q. Li, L. Chen, M. R. Gadinski, S. Zhang, G. Zhang, H. Li, A. Haque, L.-Q. Chen, T. Jackson and Q. Wang, Nature, 2015, 523, $576 і ̈ 579$

43. T. B. Adams, D. C. Sinclair and A. R. West, Adv. Mater., 2002, 14, 1321ï 1323

44. H. J. Kim, S.-M. Lee, Y.-S. Oh, Y.-H. Yang, Y. S. Lim, D. H. Yoon, C. Lee, J.-Y. Kim and R. S. Ruoff, Sci. Rep., 2014, 4, 5176

45. B. Román-Manso, E. Domingues, F. M. Figueiredo, M. Belmonte and P. Miranzo, J. Eur. Ceram. Soc., 2015, 35, 2723 Ï 2731

46. L. S. Walker, V. R. Marotto, M. A. Rafiee, N. Koratkar and E. L. Corral, ACS Nano, 2011, 5, $3182 і ̈ 3190$

47. U. G. K. Wegst, H. Bai, E. Saiz, A. P. Tomsia and R. O. Ritchie, Nat. Mater., 2015, 14, $23 \ddot{i} 36$

48. P. Ninz, R. Landfried, F. Kern and R. Gadow, J. Eur. Ceram. Soc., 2015, 35, 4031 Ï 4037

49. S. Put, J. Vleugels, O. van der Biest, C. S. Trueman and J. Huddleston, Br. Ceram. Trans., 2001, 100, $207 і ̈ 213$

50. L. Melk, M.-L. Antti and M. Anglada, Ceram. Int., 2016, 42, 5792ï 5801

51. K. Niihara, R. Morena and O. Metals, J. Mater. Sci. Lett., 1982, 1, 131 Ï 16 